Thomas Coelen, Simon Hemmerich, Hannah Jestädt, Sarah Klepp, Angela Million \& Christine Zinke

\title{
Bildungslandschaften in Campus-Form aus schulischer Perspektive
}

\section{Zusammenfassung}

Bildungslandschaften sollen informelle und formelle Bildungsprozesse ermöglichen. Die Campus-Form wird dabei vielerorts als Idealvorstellung angesehen. Sie soll eine engere Zusammenarbeit von Schulen und anderen Bildungseinrichtungen in hierfür geeigneten Architekturen und Außenräumen unterstützen. Sozialräumliche Aspekte sowie die Verknüpfung von Quartiersentwicklung und Bildungswesen spielen dabei eine wesentliche Rolle. Die Bedeutung der Campus-Form wird in diesem Artikel anhand von Interviews mit Schulakteur*innen herausgearbeitet.

Schlüsselwörter: Sozialräumliche Bildungslandschaften, Campus, Stadtentwicklung, Städtebau, Bildungswesen, Schulentwicklung, Sozialraum, Öffnung von Schule, Kommunales Bildungsmanagement

\section{Educational Landscapes in Campus Form from a School Perspective}

\begin{abstract}
Educational landscapes aim to facilitate both informal and formal educational processes. Commonly, the campus form is seen as an ideal concept. It is intended to facilitate closer cooperation between schools and other educational actors by providing adequate architectures and outdoor spaces. Socio-spatial aspects as well as the linking of neighborhood development and education play an important role. The significance of the campus will be elaborated in this article based on interviews with school actors.

Keywords: socio-spatial educational landscapes, campus, urban planning, urban design, educational system, school development, social space, open school, education management
\end{abstract}




\section{Einführung}

Gesellschaftlicher und insbesondere demografischer Wandel sowie die Erkenntnisse aus PISA 2001 führten zu einem Überdenken bisheriger Institutionen und Strukturen im Bildungswesen und tendenziell zu einer Verlagerung der Bildungskoordination und -steuerung auf die kommunale Ebene (vgl. Coelen et al., 2019, S. 38; Deutscher Städtetag, 2007). Im Zuge dieses Kommunalisierungsprozesses von Bildung (Weiß, 2011, S. 9-22) wurde auch die Weiterentwicklung und Einbeziehung integrierter Stadtentwicklungskonzepte immer wichtiger (Million et al., 2017, S. 17 f.). Daher rückten Handlungsansätze in den Vordergrund, die Stadtentwicklung und Bildungswesen integrierten - häufig in Form sogenannter sozialräumlicher Bildungslandschaften. Das von vielen beteiligten Akteur*innen häufig beschriebene Ideal ist die Campus-Form (ebd., S. 225) - eine architektonisch verdichtete Form lokaler Bildungslandschaft, der oft ein ganzheitliches Bildungsverständnis zugrunde liegt.

Dieser Artikel widmet sich nach einer Einführung in den Forschungsstand und der Definition relevanter Begriffe sowie der Erläuterung möglicher Wechselwirkungen von Sozialraum und Bildung den in der empirischen Forschung bisher erstaunlich wenig berücksichtigten schulischen Perspektiven auf Bildungslandschaften. Der Artikel nimmt dabei Bezug auf unser von der Deutschen Forschungsgemeinschaft (DFG) gefördertes Projekt „Der Campus als Leitbild und Praxis in Lokalen Bildungslandschaften“. Die hier angeführten Ergebnisse wurden aus Interviews mit schulischen Akteur*innen in Leitungsfunktionen in zwei ausgewählten Campusprojekten gewonnen. Ein nächster Projektschritt sieht vor, auch außerschulische Akteur*innen sowie Jugendliche in unsere Analyse einzubeziehen.

\section{Forschungs- und Entwicklungsstand}

Im Folgenden werden die gängigen Begriffe der Debatte skizziert. Zunächst erläutern wir das Konzept von Bildungslandschaften und gehen anschließend auf die Verbindung von Sozialraum und ganzheitlichem Bildungsverständnis ein. Abschließend betrachten wir die Besonderheiten von sozialräumlichen Bildungslandschaften, die sich in einigen Kommunen in architektonisch-städtebaulichen Formen materialisiert haben, speziell in Form von Campus.

\subsection{Bildungslandschaften}

Der Begriff der Bildungslandschaft wird vielfältig verwendet. Grundsätzlich impliziert er das „Zusammenspiel von Erziehung, Bildung und Betreuung im sozialen (Nah-) 
Raum“ (Bollweg, 2018, S. 1162). Dabei sind vier Typen beobachtbar (vgl. Berse, 2011, S. 41-44):

1) „Kooperation von Jugendhilfe und Schule“;

2) „Schule und Gestaltung von Schulentwicklung“;

3) „Lebenslanges Lernen, Weiterbildung und Wirtschaft“;

4) „Sozialer Raum als Bildungsraum“.

Sozialräumliche Bildungslandschaften in Campus-Form sind eine Spezialform der vierten Variante. Dabei steht, im Sinne Christoph Berses, der gesamte lokale Sozialraum im Fokus der pädagogischen und städtebaulichen Aktivitäten, da die „Gestaltung der sozialräumlichen Lebensbedingungen [...] Grundlage für Bildungsprozesse“ (vgl. Berse, 2011, S. 43 f.) ist.

\subsection{Sozialraum und ganzheitliches Bildungsverständnis}

Ein ganzheitliches Bildungsverständnis stellt die Bedeutung vielfältiger sowohl formeller als auch informeller Bildungsprozesse in formalen wie non-formalen Bildungssettings in den Fokus (vgl. BMFSFJ, 2005, S. 94-97). Bildung wird darin als eine Gestaltungsaufgabe und Möglichkeit zur Persönlichkeitsentwicklung insbesondere von Kindern und Jugendlichen verstanden (vgl. ebd., S. 424). Der Sozialraum ist dafür buchstäblich fundamental (vgl. Coelen, 2002, S. 201), indem dieser „ein Geflecht von Interaktionen, Institutionen und Normen enthält und ermöglicht bzw. verhindert" (Coelen, 2020, S. 1292f.). Der Sozialraum markiert in diesem Verständnis die „Bedingungen der Möglichkeit sozialer Praktiken“ (Kessl \& Reutlinger, 2013, S. 60) und so auch die Bedingungen bildungsbezogener Praxis. Bildungslandschaften erweitern damit ein schulzentriertes Bildungsverständnis (vgl. Million et al., 2017, S. 26f.). Angestrebt wird allgemein eine Verschränkung verschiedener pädagogischer, räumlicher und politischer Ermöglichungsaspekte von ganzheitlicher Bildung. Der Sozialraum bildet hierbei sowohl den Ausgangs- als auch den wesentlichen Bezugspunkt.

\subsection{Campus als besondere Form sozialräumlicher Bildungslandschaften}

Viele Akteur*innen auf den Ebenen von Bund, Ländern, Kommunalverbänden und Kommunen benennen einen Campus als gestalterisches Ideal von lokalen Bildungslandschaften (vgl. ausführlich Million et al., 2017). Dabei wird unter einem Campus eine besonders verdichtete Form von lokal gebauten Bildungslandschaften verstanden, die architektonisch und städtebaulich vor allem durch kraftvolle Gebäudevolumina, die Herausbildung qualitativ hochwertiger Freiräume und eine Nutzungsmischung gekennzeichnet ist (vgl. ebd., S. 207). Durch die Campusform sollen verschiedene Bildungsakteur*innen auf einem begrenzten Areal innerhalb eines 
Sozialraums zusammengebracht werden. Der Kooperation der verschiedenen Einrichtungen liegt eine gemeinsame pädagogische Idee bzw. ein Leitbild zugrunde. Die Verknüpfung sozialräumlicher und bildungsbezogener Aspekte durch eine architektonisch-pädagogisch durchdachte materielle Ausgestaltung (sowohl von Gebäuden als auch von Außenflächen) soll die Basis für eine engere Vernetzung verschiedener Bildungsakteur*innen und -einrichtungen bilden. Durch die räumliche Nähe der Einrichtungen sowie deren Kooperation miteinander sollen Übergänge zwischen Bildungsinstitutionen erleichtert und Zugänge zu Freizeitangeboten geschaffen werden. Dies betrifft beispielsweise Schulen, Kindertagesstätten und andere Einrichtungen der Kinder- und Jugendhilfe sowie an größeren Zielgruppen orientierte Einrichtungen wie Bibliotheken, Volkshochschulen, Schwimmbäder u.v.a.m. Somit sollen vielfältige Bildungsprozesse ermöglicht und zudem ein „Beitrag für mehr Bildungsgerechtigkeit und Teilhabechancen“" (Million, 2020, S. 1329) geleistet werden.

Schulen, vor allem solche mit Ganztagsangeboten, nehmen in Bildungslandschaften einen zentralen Platz ein. Hans-Günter Rolff spricht in diesem Zusammenhang von „horizontale[r] Schulentwicklung“ (Rolff, 2016, S. 174). Dabei „mutierte sie [die Schulentwicklung, Anm. d. Verf.] [...] zur Entwicklung von regionalen Schul- und Bildungslandschaften“ (ebd.). Dieser Prozess ist mit besonderen Herausforderungen verbunden, da bspw. Leitbilder nicht für eine einzelne Organisation wie die Schule, sondern für ein ganzes Bildungsnetzwerk innerhalb der Bildungslandschaft entwickelt und umgesetzt werden müssen. Dies erfordert ggf. Fortbildungen für die verschiedenen involvierten Professionen, um erfolgreich neue Methoden implementieren zu können, die über institutionelles Lernen hinausgehen. Schule muss sich hierfür den anderen Bildungs- und Betreuungseinrichtungen gezielt öffnen. Die von Rolff in den Fokus gerückte Ganzheitlichkeit (Rolff, 2016, S. 150-154) spielt insbesondere für Bildungscampus eine wichtige Rolle und soll bspw. durch ein Campusmanagement gewährleistet werden.

In den landespolitischen Diskussionen über Schulen als Teil von Bildungslandschaften geht es bislang jedoch hauptsächlich um die Schulgebäude, weniger um innerschulische Aspekte (vgl. Million, 2020, S. 1330). Solche Angelegenheiten der inneren Schulentwicklung nehmen wir im Folgenden in den Blick, indem wir nach der Bedeutung der Campus-Form für schulische Akteur*innen fragen.

\section{Empirische Analyse: Methodik und Ergebnisse}

Bei den folgenden Ausführungen stellen wir erste Ergebnisse aus unserem aktuell laufenden, qualitativ angelegten und von der DFG geförderten Projekt „Der Campus als Leitbild und Praxis in Lokalen Bildungslandschaften“ vor. In dieser Untersuchung werden Entwicklungen und Nutzungen von ausgewählten Campusprojekten in den 
Blick genommen, u. a. durch die Befragung verschiedener Akteurinnen gebauter Bildungslandschaften. Für die nachfolgende Analyse haben wir drei online durchgeführte Interviews (I1, I2, I3) mit Akteur*innen in Leitungspositionen aus der Sekundarstufe ausgewählt, deren Schulen auf jeweils einem der bisher untersuchten Campus verortet sind. Die zwei analysierten Campusprojekte befinden sich in unterschiedlichen Bau- und Umsetzungsphasen: Eines ist bereits seit mehreren Jahren fertiggestellt, das andere befindet sich noch in der Planungs-, Bau- und Umsetzungsphase. Daraus ergeben sich je nach Realisierungszustand unterschiedliche Perspektiven: Unterschieden wird zwischen Schilderungen zum Entstehungsprozess im Falle des Campus im Bau (I2 und I3) und erfahrungsbasierten Perspektiven im Falle des Campus im Betrieb (I1). Unter Rückgriff auf ausgewählte Zitate aus den Interviews, die mithilfe der qualitativen Inhaltsanalyse nach Kuckartz (2018) ausgewertet wurden, wird nachfolgend insbesondere auf drei Aspekte eingegangen: (1) der Campus als Chance, (2) Herausforderungen, Grenzen und Konflikte und (3) Kooperationen auf dem und durch den Campus.

\subsection{Der Campus als Chance}

Die Campus(bau)projekte werden von allen Interviewpartner*innen mit Chancen für die eigene Schule, für die Lehrkräfte und für die Schüler*innen verbunden. Außerdem werden sozialräumliche Bezüge zur nächsten Nachbarschaft und zum erweiterten Quartier hergestellt. Der Campus soll nach den Worten der Interviewten zweierlei leisten. Er soll erstens Raumressourcen im Sozialraum für schulische Themen nutzbar machen sowie zweitens zur Aufwertung und Attraktivitätssteigerung des Standortes bzw. Quartiers beitragen. Ziel ist es, Menschen und Familien an das Quartier zu binden, deren Zufriedenheit vor Ort zu steigern und Abwanderung zu verhindern. Dazu könne der Campus insofern beitragen, als dass er durch das verdichtete Angebot an Schulen sowie Freizeitmöglichkeiten und die hohe Aufenthaltsqualität durch Grünflächen und andere Freiräume ein attraktives Areal für „Begegnung“ (I2_Abs. 5), „Austausch“ (I2_Abs. 5) und „Bildung“ (I3_Abs. 16) darstellt. Bedingt durch die räumliche Nähe der verschiedenen Angebote sei der Campus ein Ort der Überschneidung verschiedener Lebensbereiche - etwa zwischen Schule, Freizeit und Beruf - und habe so das Potenzial zum zentralen Dreh- und Angelpunkt des Quartiers bzw. zu einem „Lebensort“ (I1_Abs. 8) zu werden.

Die Interviewpartner*innen des Campus im Bau betonen, dass der Campus offen und für viele Zielgruppen nutzbar sein soll. Dies sei vor allem machbar, wenn wie in einem geplanten Neubau auf dem Campus - in der architektonischen Grundidee der Gebäude eine multifunktionale Nutzung von Anfang an angelegt ist, sodass zum Beispiel „Volkshochschule, Musikschule und Schule das Gebäude nutzen können, [es] Ausstellungsflächen geben kann, [...] Musikveranstaltungen möglich sind“ 
(I3_Abs. 6). Es wird erwartet, dass ein vielfältiges Bildungsangebot die Förderung von unterschiedlichen Bildungsprozessen ermöglicht.

Mit Blick auf die Schulen und die Schulentwicklungsprozesse formulieren die Schulakteur*innen des Campus im Bau weitere Chancen hinsichtlich der Umgestaltung des Geländes und vor allem der Gestaltung der Neubauten: „Das bietet natürlich Chancen, räumlich anders zu denken, als wenn ich die alten Mauern nur umbaue" (I3_Abs. 42). Während der Planungsphase orientierten sich alle schulischen Akteur*innen sowohl an nationalen „Erfolgsmodellen“ (I2_Abs. 25), als auch an „besondere[n] Schulen“ und Campusprojekten in Europa (I1_Abs. 12). Die baulichen (Um-)Gestaltungsmöglichkeiten begünstigen wiederum pädagogisch-konzeptionelle Veränderungsprozesse innerhalb der einzelnen Schulen, so die Akteur*innen beider Campus. Diese seien auch notwendig, um Schulen zukunftsfähig zu machen, denn - so argumentiert eine der befragten Personen des im Bau befindlichen Campus - Schule allein könne „so separat ja existenziell eigentlich keinen richtigen Bestand mehr“ (I3_Abs. 4) haben.

Die kommunale Schulentwicklung im Kontext des Campus im Betrieb nahm das Bauprojekt zum Anlass, um verschiedene Schulformen zusammenzuführen und den Ausbau von Ganztagsbetreuung voranzutreiben. Förderlich seien dafür vor allem weitere Kooperationsmöglichkeiten mit benachbarten Bildungsinstitutionen in Form von AGs sowie der Neubau einer Mensa. Durch die Öffnung der Mensa für Zielgruppen über die Schüler ${ }^{*}$ innen hinaus werde diese - so die Annahme einer interviewten Person - zum Begegnungsort zwischen Generationen, Anwohner*innen und verschiedenen Gruppen von Nutzer*innen und ermögliche so Anlässe für weitere Bildungsprozesse.

Innenarchitektonisch installierte die Schule des realisierten Campus außerdem Lernlandschaften mit eigenen Arbeitsplätzen für Schüler ${ }^{*}$ innen in Kombination mit Input- und Fachräumen. Diese Umgestaltung solle den eigenen schulischen Arbeitsplatz als „kleines zweites Zuhause“ (I1_Abs. 16) etablieren, welches die Schülerinnen „individuell selbst gestalten können“ (I1_Abs. 16) (vgl. Abb. 1). 
Abb. 1: Lernlandschaft mit Arbeitsplätzen für Schülerinnen

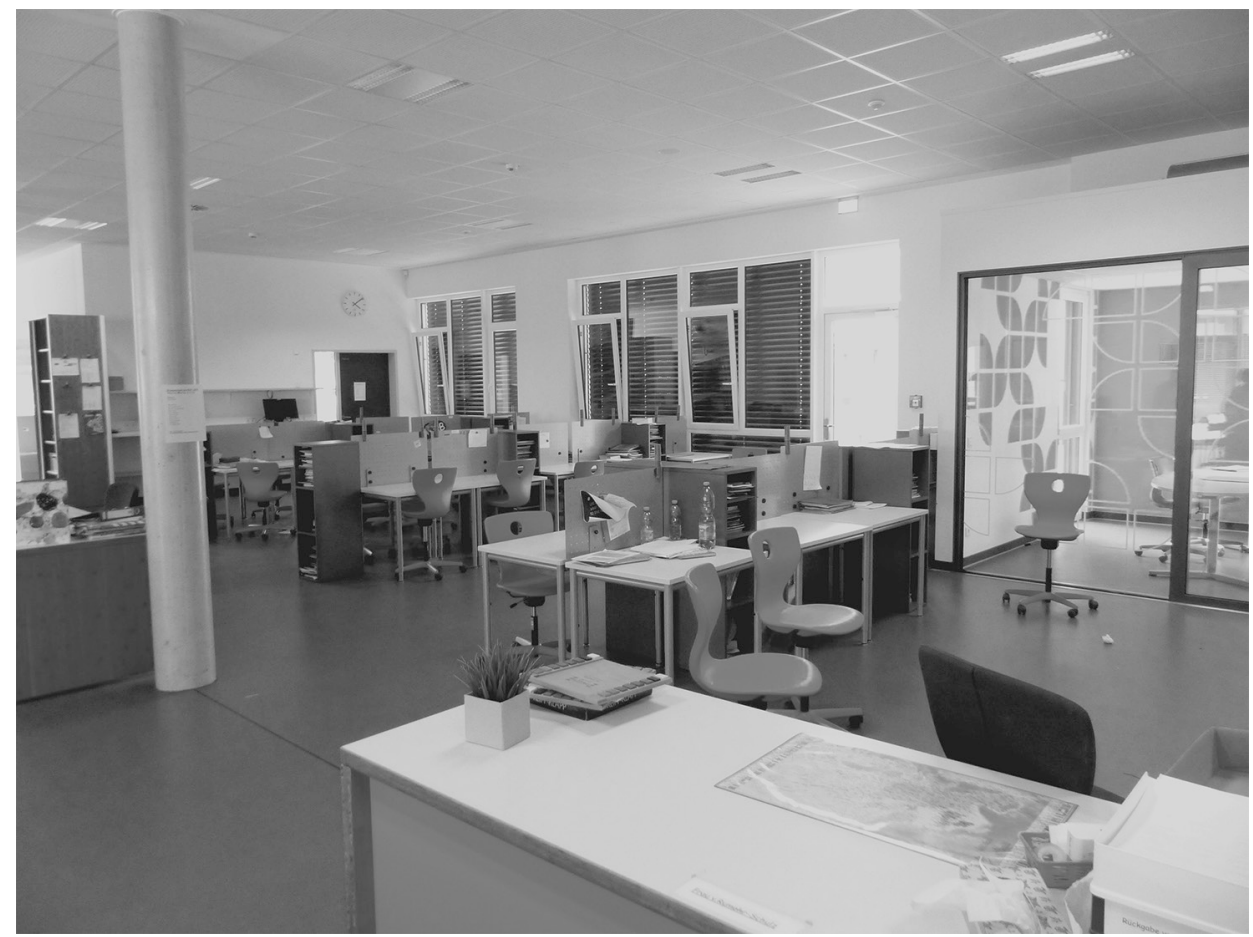

Quelle: Fotoaufnahme der Autorinnen

Die Lehrkräfte arbeiten dort nun in Teams zusammen; sie verstehen sich außerdem als „Lern-Coaches“, wobei die Beziehung zu den Schüler*innen im Fokus stehe. Ebenso wie für den Gesamt-Campus gelte auch für die Schule, dass solche konzeptionellen Veränderungsprozesse, die auf Fachkräfte und deren (Weiter-)Qualifizierung rückwirken, keineswegs mit der Fertigstellung der Gebäude abgeschlossen seien. Vielmehr sei eine kontinuierliche Weiterentwicklung wichtig, etwa durch das Einführen von Regeln zur gemeinsamen Nutzung oder durch spezielle Fortbildungen für Lehrkräfte. Zusätzliche berufliche und private Aktivitäten, wie die Nutzung von Sportkursen durch das Kollegium, würden sich durch die persönlichen Kontakte auf dem Campus ergeben „dadurch, dass man sich eben auf dem Campus auch begegnet [und] dann rankommt sozusagen“ (I1_Abs. 48). Der Campus bietet durch die Kooperation mit vielfältigen Bildungspartner*innen also neben beruflichen auch weitere Chancen für Lehrkräfte.

Neben Fragen der Schulentwicklung und architektonischen Gestaltung von Lernräumen im Allgemeinen werden gerade in der konzeptionellen Phase des Campus im Bau auch subjektorientierte pädagogische sowie bildungspolitische Ziele formuliert, die mit dem Campus verbunden werden, wie Bildungsgerechtigkeit: 
„[E]s geht schon darum zu gucken, gerade eben auch in diesem Bereich hier, dass es ja doch einen gewissen Anteil von Jugendlichen oder Kindern gibt, die eben doch relativ bildungsfern sind [...]. Wie finde ich einen vernünftigen Beruf? [...] Und das zu vermitteln mit allen Partnern auf dem Campus, glaube ich, ist ein ganz wichtiger Aspekt, was die sozialökonomische oder die soziale Entwicklung des Gebietes auch mit anspricht [...]“(I3_Abs. 20).

Die Interviews zeigen, dass von der Campus-Form auch pädagogisch relevante Erträge erhofft werden, die zugleich als ein Beitrag zur Quartiers- und Stadtentwicklung verstanden werden.

\subsection{Herausforderungen, Grenzen und Konflikte}

Die Befragten beider Standorte berichteten, dass die baulich-räumliche Offenheit des Geländes sowohl in der Planungsphase als auch im Betrieb neben den bereits beschriebenen Vorteilen auch Konfliktpotenziale bereithält. Mit Blick auf den gesamten Campus diskutieren die Interviewpartner*innen des Campus im Bau vor allem über die Grenzen bei der Umsetzung von Baumaßnahmen, bedingt durch den Bestandsschutz von Bäumen und Gebäuden. Ferner wird über $\mathrm{Zu}$ - und Übergänge des Campus in Form von räumlichen Barrieren und Umfriedungen, wie etwa Zäunen, verhandelt. Dabei werden in Bezug auf den Leitgedanken des offenen Campus Herausforderungen und Ambivalenzen im Planungs- und Umsetzungsprozess deutlich. Einerseits stünden solche Barrieren (vgl. Abb. 2) dem Leitgedanken entgegen,

„dass man im Prinzip eigentlich, ich sage es mal ganz extrem, zu jeder Tages- und Nachtzeit über das Gelände gehen kann [...]. Dass man also nicht ständig von Zäunen umgeben ist, sondern da entsprechende Freiräume schaffen kann“" (I3_ Abs. 6).

Andererseits sehen die Schulakteur*innen die Schaffung von räumlichen Barrieren auch als notwendig an, um unzulässiges Nutzungsverhalten - genannt werden Vandalismus und Müll - einzuschränken und um „Schutzbedürfnisse, erstmal für die kleineren Kinder, [...] sicher[zu]stellen“" (I3_Abs. 6). 
Abb. 2: Räumliche Barrieren in Form von Umzäunungen auf Bildungslandschaften in Campus-Form

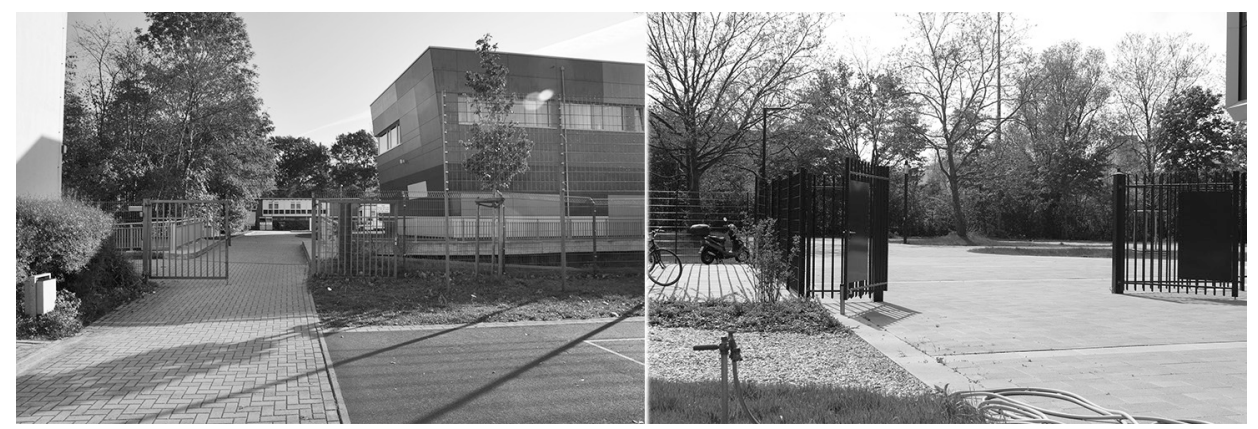

Quelle: Fotoaufnahme der Autorinnen

Ferner heben die Schulakteur*innen Herausforderungen sowohl für die Lehrkräfte als auch für die Schüler*innen während der Bauphasen hervor: Durch die langwierigen Baumaßnahmen müsse teilweise jahrelang in "provisorischen Räumen“ gelernt werden (I3_Abs. 42), welche häufig bedingt durch Lärm und Schmutz wenig lernförderlich sind (vgl. Abb. 3).

Abb. 3: Baustellensituation auf einem Campus

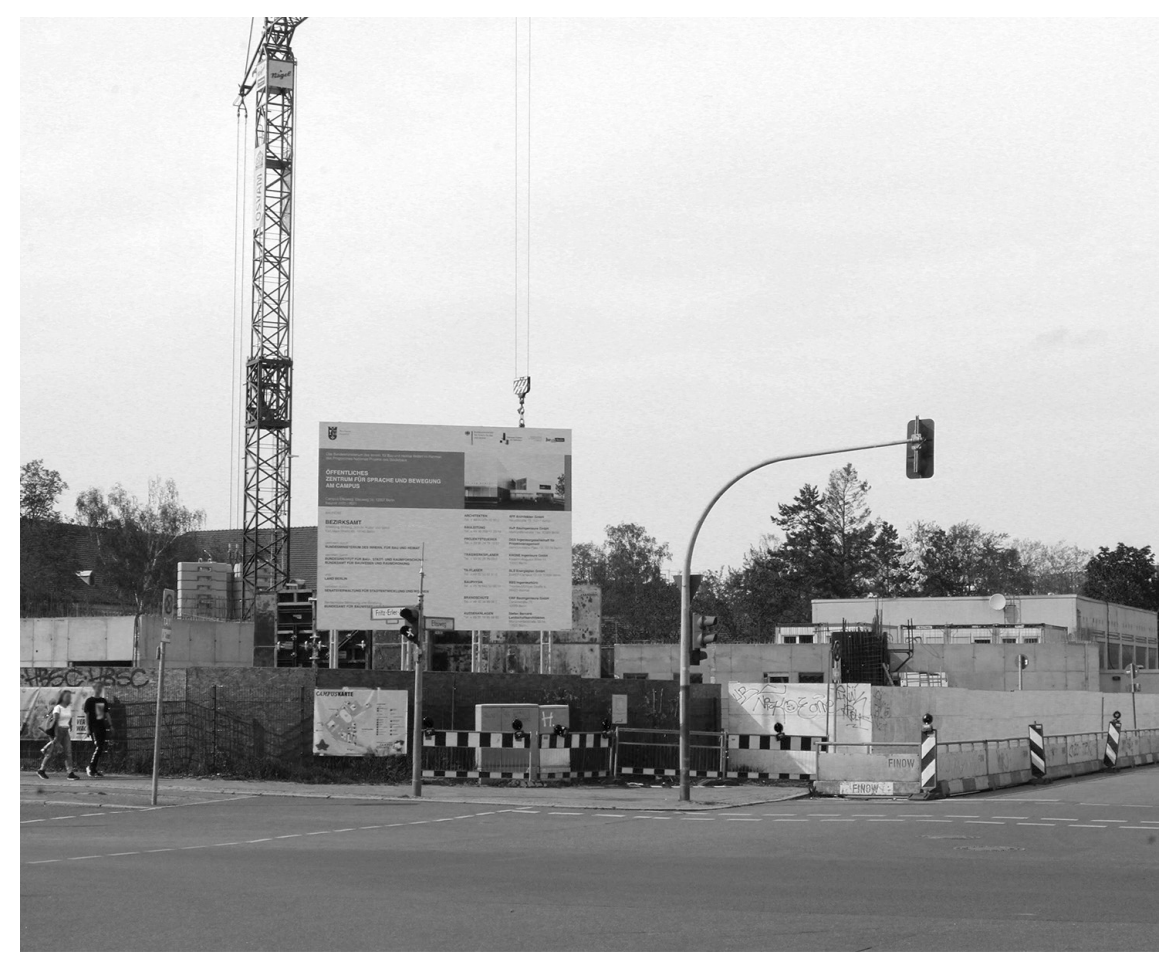

Quelle: Fotoaufnahme der Autor ${ }^{\star}$ innen 
$\mathrm{Zu}$ Ärger bei den schulischen Akteur*innen des Campus im Bau führt vor allem der von Besucher*innengruppen in den Abendstunden zurückgelassene Müll auf dem Schulgelände, der dann vor Schulbeginn auf Eigeninitiative der Schule entfernt werden müsse (I2_Abs. 8, 48). Die Nutzung des Geländes in den Abendstunden stellt auch für den Campus im Betrieb eine Herausforderung dar (I1_Abs. 56).

Weiteres Konfliktpotenzial wird in den campusbezogenen Aufgaben gesehen: Die Interviewpartner*innen, deren Campus sich aktuell in der Bau- und Konzeptionsphase befindet, befürchten einen erheblichen Mehraufwand durch zusätzliche campusbedingte Aufgaben in Bezug auf Inhalt und Organisation, z. B. Planungstreffen und Vernetzungsaktivitäten. Diese Bedenken erschweren es zum Teil auch, die Lehrkräfte für die Campusidee zu begeistern (I2_Abs. 18). Außerdem könnten die zusätzlichen Aufgaben zu einer „Überforderung [beitragen], weil Schule immer mit ihrem Alltag schon sehr stark ausgelastet ist“ (I2_Abs. 21). Zudem seien jene Aufgaben „in keinster Weise [...] in der Arbeitsbeschreibung" (I3_Abs. 28) der Akteur*innen verankert. In diesem Zusammenhang formuliert eine befragte Person, dass sie „als allererstes [...] für diese Schule verantwortlich“ sei und „der Campus ist [für sie] second“ (I2_Abs. 31). Hingegen kommen Klagen bezüglich zeitlicher und personeller Ressourcen in den Interviews mit den Akteur*innen auf dem fertiggestellten Campus nur am Rande zur Sprache. Rückblickend nennt die befragte Person hier vor allem, dass mit den konzeptionellen Veränderungen Organisations- und Personalentwicklungsprozesse verbunden seien, die Hürden bereithielten. „Reibereien“ (I1_Abs. 6) seien etwa durch die anfängliche Skepsis von einzelnen Lehrkräften und durch veränderte Arbeitsweisen entstanden:

„Wir waren 50, 60 Einzelkämpfer und mussten plötzlich in Teams gehen. [...] und dann weiß ich noch genau da: ,wer könnte sich denn vorstellen, in ein Team zu gehen, da gingen genau sechs Finger hoch [...] mehr waren es nicht" (I1_Abs. 6).

An der Zusammenarbeit in Teams sei „immer weitergefeilt und gearbeitet“ (I1_ Abs. 6) worden, etwa mithilfe von Supervisionen und Weiterbildungen. Mittlerweile funktioniere die Zusammenarbeit „ziemlich gut“ (I1_Abs. 6).

Die Schulakteur*innen des im Bau befindlichen Campus erläutern, dass bei der $\mathrm{Zu}$ sammenarbeit mit außerschulischen Akteurinnen im Rahmen der Campusprojekte gelegentlich Themen aufkämen, die für die Schule und die Schulentwicklung wenig relevant seien bzw. denen "unterschiedliche Wichtungen“ (I3_Abs. 33) beigemessen würden. Es ginge jedoch nicht nur darum, die eigene Rolle als Schule auf dem Campus zu finden, sondern auch um das Ziel, gemeinsam als „Campus hier, als organische Einheit“ (I3_Abs. 72) zusammenzuwachsen. Unterschiedliche Verwaltungszuständigkeiten für die verschiedenen Einrichtungen auf dem Campus ebenso wie Gehaltsdifferenzen würden diesen Prozess allerdings erschweren (I1_Abs. 6; I2_ Abs. 16; I3_Abs. 33). Insgesamt sei aus schulischer Perspektive für das Gelingen der 
Campusprojekte die Unterstützung und das Engagement von Schulträgern, Schulleitungen und Lehrkräften gleichermaßen wichtig.

\subsection{Kooperationen auf dem und durch den Campus}

Aus der Perspektive der Befragten ermöglichen Bildungslandschaften in CampusForm - bedingt durch die räumliche Nähe verschiedener Bildungspartner*innen niedrigschwellige und vielfältige Anlässe für Kooperationen. Ziel sei es, durch Kooperationen innerhalb solcher Bildungslandschaften - so die Befragten beider Campus - Bildungsübergänge zu erleichtern, eine „vorbildliche Bildungskette“ (I2_Abs. 5) zu ermöglichen bzw. die „Übergangsqualität“ (I3_Abs. 12) gemeinsam weiterzuentwickeln, z. B. beim „Übergang von der Kita zur Schule“ (I3_Abs. 12), beim Übergang auf weiterführende Schulen, beim Nachholen von Schulabschlüssen oder bei der Entwicklung beruflicher Perspektiven. Betont wird dabei, „Schülerinnen und Schüler anschlussfähig machen“ (I1_Abs. 12) zu wollen, nicht nur hinsichtlich der Bildungsabschlüsse, sondern damit sie „selbstständige eigenverantwortliche Menschen“ (I1_Abs. 12) werden.

Die Akteur ${ }^{\star}$ innen des Campus im Bau erläutern, dass Übergänge zwischen Schulen und anderen Institutionen, insbesondere „Von unten nach oben“ (I2_Abs. 41) - also von Schüler*innen der Sekundarstufe I (v. a. Haupt- und Realschulen) in die Sekundarstufe II - weiterhin problematisch seien, da die Anforderungen der einzelnen Institutionen divergieren (I2_Abs. 19). Die Campus-Form könne jedoch die Übergänge zwischen den verschiedenen Institutionen räumlich unterstützen, etwa durch eine attraktive Freiraumgestaltung oder durch das städtebauliche Nebeneinander von Gebäuden. So haben die Schüler*innen die Möglichkeit, schulische und außerschulische Angebote im Tagesverlauf „fließend“ (I1_Abs. 22) auf einem Gelände zu nutzen. Gleichzeitig kann die Campus-Form und -gestaltung als Mittel dienen, Kontinuität im biografischen Verlauf herzustellen und widerzuspiegeln. Denn die Schülerinnen könnten den Campus auch im Laufe ihrer Biografie durch die erleichterten Übergänge zwischen Schulformen und anderen Institutionen niedrigschwelliger nutzen und sich den Campus nicht nur räumlich, sondern eben auch bildungsbiografisch aneignen (I3_Abs. 13, 19).

Dazu blicken die Akteur*innen der beiden Campus auf ein breites Netz an Kooperationspartner*innen innerhalb und außerhalb der Bildungslandschaft, die unterschiedliche Angebote für verschiedene Zielgruppen vom Kleinkind bis zum Erwachsenen bereitstellen. Das Kooperationsangebot sei vielfältig; mal handele es sich um einmalige projektbezogene Tätigkeiten, mal um dauerhafte Kooperationen, mal mit starkem Schulbezug oder auch thematisch weiterführend. Unterstützend sei dabei ein gemeinsames Leitbild, das „im pädagogischen Konzept oder der inhaltlichen Ausrichtung von allen Einrichtungen mitgetragen“ (I3_Abs. 31) werde. Vorteilhaft sei 
außerdem die räumliche Nähe zwischen den Institutionen auf dem Campus. Dies ermögliche einen persönlichen Kontakt der Akteur*innen, der es zulasse, Themen „auf dem kurzen Wege und kurzem Draht“ (I1_Abs. 55) sowie „schnell und unkompliziert“ (I2_Abs. 55) zu besprechen. So falle es leichter, den Campusgedanken „mit im Kopf [zu haben] in seinem täglichen Handeln“ (I3_Abs. 57). Ferner könnten so auch Ressourcen wie Räumlichkeiten oder Equipment gebündelt und gemeinschaftlich genutzt werden (I3_Abs. 7). Die bauliche Struktur sollte dabei der pädagogischen Ausrichtung entsprechen.

Gelingende Kooperationen - so eine befragte Person des im Betrieb befindlichen Campus - könne es nur geben, wenn alle Partner*innen gewillt seien zu kooperieren und zeitliche, personelle und finanzielle Ressourcen zur Verfügung stünden. Hilfreich seien vor allem regelmäßige Treffen der Akteur*innen und ein federführendes Campusmanagement, das Kooperationen anregt und koordiniert. Das Campusmanagement solle dabei vergleichbare Aufgaben übernehmen wie z. B. Transferagenturen im kommunalen Bildungsmanagement, jedoch nicht in gleicher Weise datenbasiert, sondern eher auf bilateralen Austausch ausgelegt und lokal stärker begrenzt. Beim untersuchten Campus im Bau übernimmt diese Funktion momentan noch überwiegend die zuständige Stadtverwaltung, wobei der Fokus hier auf baulichen Aspekten liegt. Ein Campusmanagement und eine Netzwerkkoordination werden derzeit eingerichtet.

\section{$4 \quad$ Fazit}

Unsere Untersuchung zeigt, dass Bildungslandschaften in Campus-Form aus schulischer Perspektive vielfältige Potenziale für Bildungs- und Entwicklungsprozesse bieten - sowohl für die Schüler*innen als auch für den Sozialraum. So schafft die neue räumlich-materielle Ausgestaltung von Bildungslandschaften in Campus-Form Anlässe für zukunftsorientierte Schulentwicklungsprozesse und ein konzeptionelles Überdenken bisheriger schulischer Praxen. Als besondere Ressource sehen die Befragten die vielfältigen und niedrigschwelligen Kooperationsmöglichkeiten zwischen verschiedenen schulischen und außerschulischen Akteur*innen, die durch räumliche Nähe auf dem Campus befördert werden. Für Schüler*innen ergeben sich aus Sicht der Interviewten vor allem Chancen bzgl. der Übergangsgestaltung zwischen verschiedenen Schulformen und anderen Institutionen. Wenngleich Bildungslandschaften in Campus-Form Übergänge räumlich und biografisch vereinfachen, bedeute dies jedoch nicht eine Reduktion der schulspezifischen Anforderungen.

In einigen Bereichen berichten die befragten Akteur*innen von ähnlichen Erfahrungen, dazu zählen: 
- die gemeinsame Nutzung von Ressourcen durch unterschiedliche Akteurinnen;

- die Möglichkeit, konzeptionelle Veränderungsprozesse zu initiieren;

- die Schaffung neuer Kooperationsmöglichkeiten;

- die Durchführung baulicher Verbesserungen an Gebäuden und Außenflächen sowie

- die Steigerung der Attraktivität des Quartiers.

Diese Gemeinsamkeiten sind mit entsprechenden Konflikten verbunden, wie auch mit der Frage nach der Öffnung des Campus für die Nachbarschaft oder nach dem Einbezug aller Akteur*innen in den Campus-Gedanken. Ein gemeinsames pädagogisches Leitbild und ein räumliches Konzept scheinen hier bei der Auseinandersetzung mit den Herausforderungen zu helfen. Dabei sind je nach Realisierungsgrad des Campus unterschiedliche Aspekte für die Befragten von besonderer Relevanz - ein Zeichen dafür, dass Bildungslandschaften einem fortlaufenden Entwicklungsprozess unterliegen.

Bedeutung erhält die Campus-Form für schulische Akteurinnen somit auf verschiedenen Ebenen und mit unterschiedlichen Gewichtungen: Im schulischen Alltag scheint der Campus insbesondere bei den Befragten des Campus im Bau eher nebensächlich zu sein, dennoch ergeben sich durch den Bau neue Aufgaben, die auch mit Chancen verbunden seien: Chancen für Veränderungen des Sozialraums und für die Konzeption der Schulen sowie Chancen für Schüler*innen und für Lehrkräfte (sowohl in beruflicher als auch in privater Hinsicht). Die Wahrnehmung des Campus durch schulische Akteur*innen ist somit insgesamt als ambivalent zu bezeichnen: Insbesondere in der Entstehungsphase scheinen die Konflikte verstärkt im Vordergrund zu stehen. Zugleich werden aber auch Potenziale benannt, die über bisherige Einzelschulformen hinausgehen. Aus Sicht von Schulakteur*innen wird die Schule durch ihre Verortung in gebauten Bildungslandschaften bislang (nur) ergänzt; aus unserer Sicht ist der Campus für Schulen (noch) kein Fundament, sondern (bisher) ein Additum.

\section{Literatur und Internetquellen}

Berse, C. (2011). Kommunale Bildungslandschaften - Eine geeignete Konzeptfigur für die Förderung mehrdimensionaler Bildung? In P. Bollweg \& H.-U. Otto (Hrsg.), Räume flexibler Bildung (S. 39-50). VS.

Bollweg, P. (2018). Bildungslandschaften. In K. Böllert (Hrsg.), Kompendium Kinder- und Jugendhilfe (S. 1161-1180). Springer Fachmedien. https://doi.org/10.1007/978-3-53119096-9_55

BMFSFJ (Bundesministerium für Familie, Senioren, Frauen und Jugend). (2005). 12. Kinderund Jugendbericht. Bericht über die Lebenssituation junger Menschen und die Leistungen der Kinder- und Jugendhilfe in Deutschland. BMFSFJ. https://www.familienatlas.de/sites/fama/files/atoms/files/12._kinder-_und_jugendbericht_bmfsfj.pdf

Coelen, T. (2002). Kommunale Jugendbildung. Peter Lang. 
Coelen, T. (2020). Kommunale Jugendbildung. In P. Bollweg, J. Buchna, T. Coelen \& H.-U. Otto (Hrsg.), Handbuch Ganztagsbildung (Band 2) (S. 1287-1298). Springer Fachmedien. https://doi.org/10.1007/978-3-658-23230-6_95

Coelen, T., Heinrich, A. J., \& Million, A. (2019). Local Educational Landscapes in Germany. Interfaces and Interlacings Between Education and Urban Development. In H. Jahnke, C. Kramer \& P. Meusburger (Hrsg.), Geographies of Schooling. Knowledge and Space (S. 35-53). VS. https://doi.org/10.1007/978-3-030-18799-6_3

Deutscher Städtetag (2007). Aachener Erklärung des Deutschen Städtetages anlässlich des Kongresses „Bildung in der Stadt“ am 22./23. November 2007. https://www.staedtetag. de/files/dst/docs/Dezernat-3/Archiv/aachener-erklaerung-2007.pdf

Kessl, F., \& Reutlinger, C. (2013). Jugend und Sozialraum. In Y. Kaiser, M. Spenn, M. Freitag, T. Rauschenbach \& M. Corsa (Hrsg.), Handbuch Jugend. Evangelische Perspektiven (S. 60-65). Verlag Barbara Budrich. https://doi.org/10.2307/j.ctvddzsbn.11

Kuckartz, U. (2018). Qualitative Inhaltsanalyse. Methoden, Praxis, Computerunterstützung. Beltz Juventa.

Million, A. (2020). Stadtentwicklung und Bildung. In P. Bollweg, J. Buchna, T. Coelen \& H.-U. Otto (Hrsg.), Handbuch Ganztagsbildung (Band 2) (S. 1323-1335). Springer Fachmedien. https://doi.org/10.1007/978-3-658-23230-6_98

Million, A., Coelen, T., Heinrich, A. J., Loth, C., \& Somborski, I. (2017). Gebaute Bildungslandschaften. jovis Verlag.

Rolff, H.-G. (2016). Schulentwicklung kompakt - Modelle, Instrumente, Perspektiven. Beltz.

Weiß, W. W. (2011). Kommunale Bildungslandschaften. Chancen, Risiken und Perspektiven. Juventa.

Thomas Coelen, Prof. Dr., geb. 1966, Erziehungswissenschaftler, Professor für Jugendbildung, Sozialisation und Lebenslaufforschung an der an der Fakultät Bildung . Architektur · Künste der Universität Siegen.

E-Mail: thomas.coelen@uni-siegen.de

Simon Hemmerich, M. A. Bildung und Soziale Arbeit, geb. 1990, Wissenschaftlicher Mitarbeiter an der Fakultät Bildung · Architektur · Künste der Universität Siegen.

E-Mail: simon.hemmerich@uni-siegen.de

Hannah Jestädt, M. A. Erziehungs- und Bildungswissenschaft, geb. 1995, Wissenschaftliche Mitarbeiterin an der Fakultät Bildung · Architektur - Künste der Universität Siegen.

E-Mail: hannah.jestaedt@uni-siegen.de

Korrespondenzadresse: Universität Siegen, Fakultät Bildung · Architektur - Künste, Adolf-Reichwein-Straße 2, 57076 Siegen,

Sarah Klepp, M. A. Historische Urbanistik, geb. 1984, Wissenschaftliche Mitarbeiterin an der Technischen Universität Berlin, Fakultät VI Planen - Bauen - Umwelt, Institut für Stadt- und Regionalplanung (ISR), Fachgebiet Städtebau und Siedlungswesen.

E-Mail: s.klepp@tu-berlin.de 
Angela Million, Prof. Dr.-Ing., Stadtplanerin und Städtebauerin, geb. 1974, Professorin und Fachgebietsleiterin Städtebau und Siedlungswesen an der Technischen Universität Berlin, Fakultät VI Planen - Bauen - Umwelt, Institut für Stadt- und Regionalplanung (ISR).

E-Mail: million@tu-berlin.de

Korrespondenzadresse: Technische Universität Berlin, Fakultät VI Planen - Bauen - Umwelt, Institut für Stadt- und Regionalplanung (ISR), Fachgebiet Städtebau und Siedlungswesen, Hardenbergstr. 40A, 10623 Berlin

Christine Zinke, M. A. Bildung und Soziale Arbeit, geb. 1990, Wissenschaftliche Mitarbeiterin an der Fakultät Bildung · Architektur · Künste der Universität Siegen E-Mail: christine.zinke@uni-siegen.de

Korrespondenzadresse: Universität Siegen, Fakultät Bildung · Architektur · Künste, Adolf-Reichwein-Straße 2, 57076 Siegen 